\title{
A systematic review of the literature assessing the outcomes of stapled haemorrhoidopexy versus open haemorrhoidectomy
}

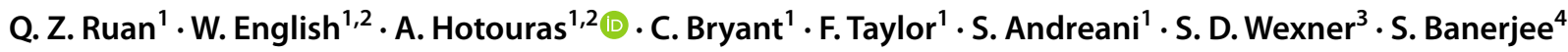

Received: 30 June 2020 / Accepted: 16 July 2020 / Published online: 24 October 2020

(c) The Author(s) 2020

\begin{abstract}
Background Symptomatic haemorrhoids affect a large number of patients throughout the world. The aim of this systematic review was to compare the surgical outcomes of stapled haemorrhoidopexy $(\mathrm{SH})$ versus open haemorrhoidectomy $(\mathrm{OH})$ over a 20 -year period.

Methods Randomized controlled trials published between January 1998 and January 2019 were extracted from Pubmed using defined search criteria. Study characteristics and outcomes in the form of short-term and long-term complications of the two techniques were analyzed. Any changes in trend of outcomes over time were assessed by comparing article groups 1998-2008 and 2009-2019.

Results Twenty-nine and 9 relevant articles were extracted for the 1998-2008 (period 1) and 2009-2019 (period 2) cohorts, respectively. Over the two time periods, SH was found to be a safe procedure, associated with statistically reduced operative time (in 13/21 studies during period 1 and in 3/8 studies during period 2), statistically less intraoperative bleeding (3/7 studies in period 1 and 1/1 study in period 2) and consistently less early postoperative pain on the visual analogue scale (12/15 studies in period 1 and $4 / 5$ studies in period 2) resulting in shorter hospital stay (12/20 studies in period 1 and 2/2 studies in period 2) at the expense of a higher cost. In the longer term, although chronic pain in $\mathrm{SH}$ and $\mathrm{OH}$ patents is comparable, patient satisfaction with SH may decline with time and at 2-year follow-up $\mathrm{OH}$ appeared to be associated with greater patient satisfaction.
\end{abstract}

Conclusions SH appears to be safe with potential advantages, at least in the short term, but the evidence is lacking at the moment to suggest its routine use in clinical practice.

Keywords Haemorrhoidectomy $\cdot$ Stapled $\cdot$ Open $\cdot$ Outcomes

\section{Introduction}

Symptomatic haemorrhoids account for approximately 3.3 million outpatient encounters annually in the United States [1], while up to $37 \%$ of the general population in the United Kingdom may be affected by the same disease process [2].

A. Hotouras

alex007@ doctors.org.uk

1 Whipps Cross University Hospital, Barts Health NHS Trust, London, UK

2 National Bowel Research Centre, Blizard Institute, QMUL, 2 Newark Street, London E1 2AT, UK

3 Cleveland Clinic Florida, Fort Lauderdale, FL, USA

4 Barking, Havering and Redbridge University Hospitals NHS Trust, Romford, UK
Grade III and IV haemorrhoidal disease (Goligher classification) responds more favourably to surgical treatment [3]. Traditional open haemorrhoidectomy $(\mathrm{OH})$ is still the gold standard operation but it is associated with significant postoperative pain and a small risk of injury to the anal sphincter complex $[4,4]$. Novel surgical procedures such as the haemorrhoidal artery ligation operation (HALO) and stapled haemorrhoidopexy $(\mathrm{SH})$ have been increasingly used in recent years. However, robust evidence strongly supportive of a specific technique is lacking. The introduction of $\mathrm{SH}$ in 1998 promptly caught the interest of colorectal surgeons. The technique has been used mostly in North America and European countries. The United Kingdom has yet to adopt this procedure on a significant scale. The aim of this systematic review was to assess the surgical outcomes of this procedure in comparison to $\mathrm{OH}$ over a 20 -year period (since 
the introduction of SH in 1998) and assess changes in its safety profile to the present day.

\section{Materials and methods}

\section{Search strategy and study selection}

The PubMed database was searched for relevant studies published between January 1998 and January 2019. The search criteria 'staple* AND haemorrhoid* OR hemorrhoid' were broadly used and a series of rigid inclusion criteria were subsequently applied. A study was deemed suitable for inclusion if the publication [1] was a randomized controlled trial (RCT) [2], compared at least two surgical methods of haemorrhoidal management with mandatory inclusion of $\mathrm{OH}$ [3], involved human subjects, and [4] was written in English. Two independent reviewers (QZR and AH) used the above-mentioned inclusion criteria for all research papers derived from the search. Studies were included after titles and abstracts were evaluated for suitability. Articles without abstracts were excluded. Full-text versions were then acquired. In the event of disagreement, a consensus method was used amongst the two reviewers The review was conducted in accordance with the guidelines set out in the "Preferred Reporting Items for Systematic Reviews and Meta-Analyses" (PRISMA) statement [6]. Collectively, selected articles were subcategorized into two classes by year (1998-2008; 2009-2019) for data interpretation and subsequent comparison.

\section{Data extraction and outcome measures}

Data extracted included study characteristics (study objective, type of study, methods of analysis) and outcomes (immediate complications, long-term complications, and overall qualitative conclusion). The derivative qualitative conclusions were defined by the positions represented by the most number of articles for that category (SH superior, $\mathrm{SH}$ similar to $\mathrm{OH}, \mathrm{SH}$ inferior). If there were equal numbers of articles for opposing positions, the position with the most number of articles with supporting $p$ values was taken as the overall qualitative conclusion. Only statistically significant $p$ values were taken as the benchmark when assessing validity of study conclusions. Some studies did not calculate statistical significance when comparing the above parameters and chose to qualitatively summarize their data. We have made it a point to label their $p$ values 'undefined', but their observations continued to be acknowledged during our analyses.

\section{Statistical analysis}

GraphPad Prism 7.0 (GraphPad Software, Inc. La Jolla, CA, USA) was used for all statistical calculations in this paper. Student's $t$ test and the Mann-Whitney test were employed to compare continuous trends in complications across the two chronological classes (1998-2008 and 2009-2019), while Fisher's exact test was used to determine differences in categorical outcomes. A $p$ value $<0.05$ was considered statistically significant.

\section{Results}

In total, 1716 articles were initially identified by the search on 12th January 2019. After abstract screening and exclusion, a total of 38 articles met inclusion criteria for further analysis (Fig. 1).

Twenty-nine [7-35] and 9 articles [36-44] were selected for the chronological categories of 1998-2008 and 2009-2019, respectively. The mean number of patients per study was $197 \pm 80$ (range 22-3000 patients). Patients were followed-up for a mean of $76.5 \pm 14.4$ weeks (range 4.4-365.4) weeks. Randomisation was conducted prior to treatment for the majority of the studies (24 of 38). Twentythree of the studies demonstrated that there was no statistically significant difference in demographics between the patient groups being compared. Basic study characteristics are demonstrated in Tables 1, 2.

Fourteen of the trials included procedures on grades III and IV haemorrhoids only. The remaining trials included interventions on grades II, III and IV haemorrhoids in various permutations. Ten articles did not ascertain the types of haemorrhoids treated. All patients had previously failed non-operative management. Specifically for $\mathrm{SH}$, the majority of studies used the "Procedure for Prolapse and Haemorrhoids" (PPH) stapler (22 of 38). All studies assessed similar outcomes, classifying them largely into immediate complications, long-term complications, recurrences and patient satisfaction. Three of the trials performed cost analyses.

For comparisons against $\mathrm{SH}$, alternative operative interventions considered $\mathrm{OH}$ Harmonic scalpel haemorrhoidectomy (HSH) and Ligasure haemorrhoidectomy (LH).

Studies performed from 2009 to 2019 incorporated larger study populations $(561 \pm 314$ vs $84 \pm 16$, $p=0.009)$ and trended towards a longer follow-up period $(103.4 \pm 30.2$ weeks vs $68.1 \pm 16.4$ weeks, $p=0.3)$ compared to the period of 1998-2008. The likelihood of the RCTs having formal descriptions of comparable patient demographics $(p>0.9)$ and study randomization $(p=0.44)$ were similar over the two time periods, as were studies to include 
Fig. 1 PRISMA study inclusion flowchart

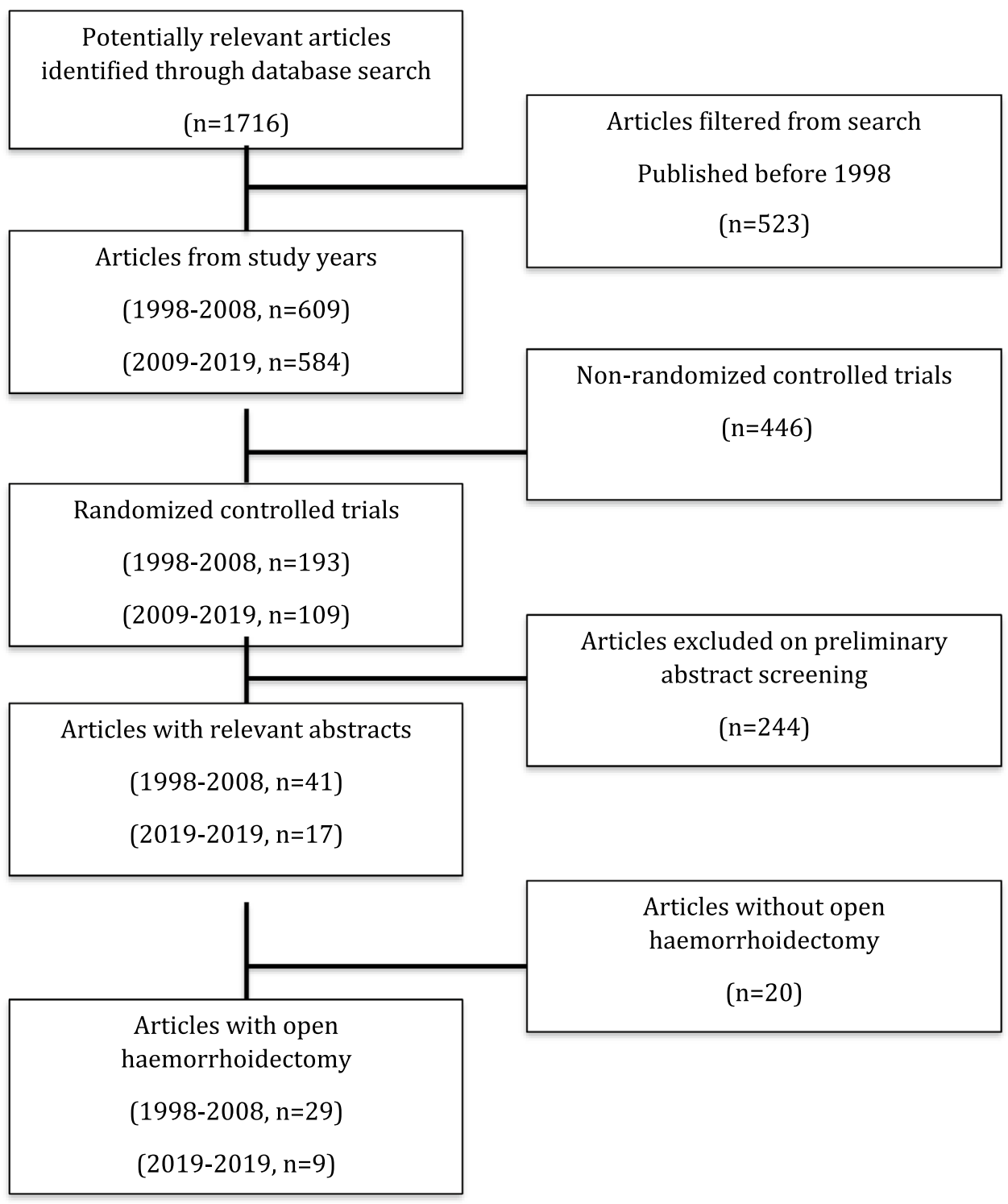

a section on cost analyses comparing the methods of haemorrhoidectomy $(p>0.9)$.

\section{Immediate complications}

Multiple immediate complications and outcomes were assessed among various operative groups including prolonged operative time, intraoperative blood loss, postoperative burning, postoperative bleeding, urinary retention, length of hospital stay and wound infection.

From 1998 to 2008, a total of 13 out of 21 studies reported a significantly shorter operative duration for $\mathrm{SH}$ than for $\mathrm{OH}$ in comparison to just 2 which stated otherwise (Table 3). Similarly from 2009 to 2019 (Table 4), five of eight studies reported similar findings (3 to statistical significance) with just one arguing the reverse (that $\mathrm{SH}$ requires longer operative time). Immediate postoperative bleeding was similar between $\mathrm{SH}$ and $\mathrm{OH}$ in both decade-long categories but more studies revealed $\mathrm{SH}$ to be favourable in reducing urinary retention in the 1998-2008 group (10 of 17 vs 4 of 17), although this only reached significance in 1 study, as well as the 2009-2019 group (3 of 6 vs 0 of 6) 2 studies reaching significance. Total length of hospital stay of $\mathrm{SH}$ patients was shorter in both groups, with 14 of 20 (10 reaching significance) (1998-2008) and 3 of 6 (2 reaching significance) (2009-2019) articles demonstrating shorter hospital stay over the last 20 years. The above data are summarized in Tables 3, 4.

A total of 20 studies used a visual analogue scale (VAS) to assess postoperative pain. Earlier studies (1998-2008) tended to measure postoperative pain over the short term (hours to days), with only one study exploring pain at the 4-week mark [34]. These studies were consistent in demonstrating significantly lower VAS scores in the SH group, 


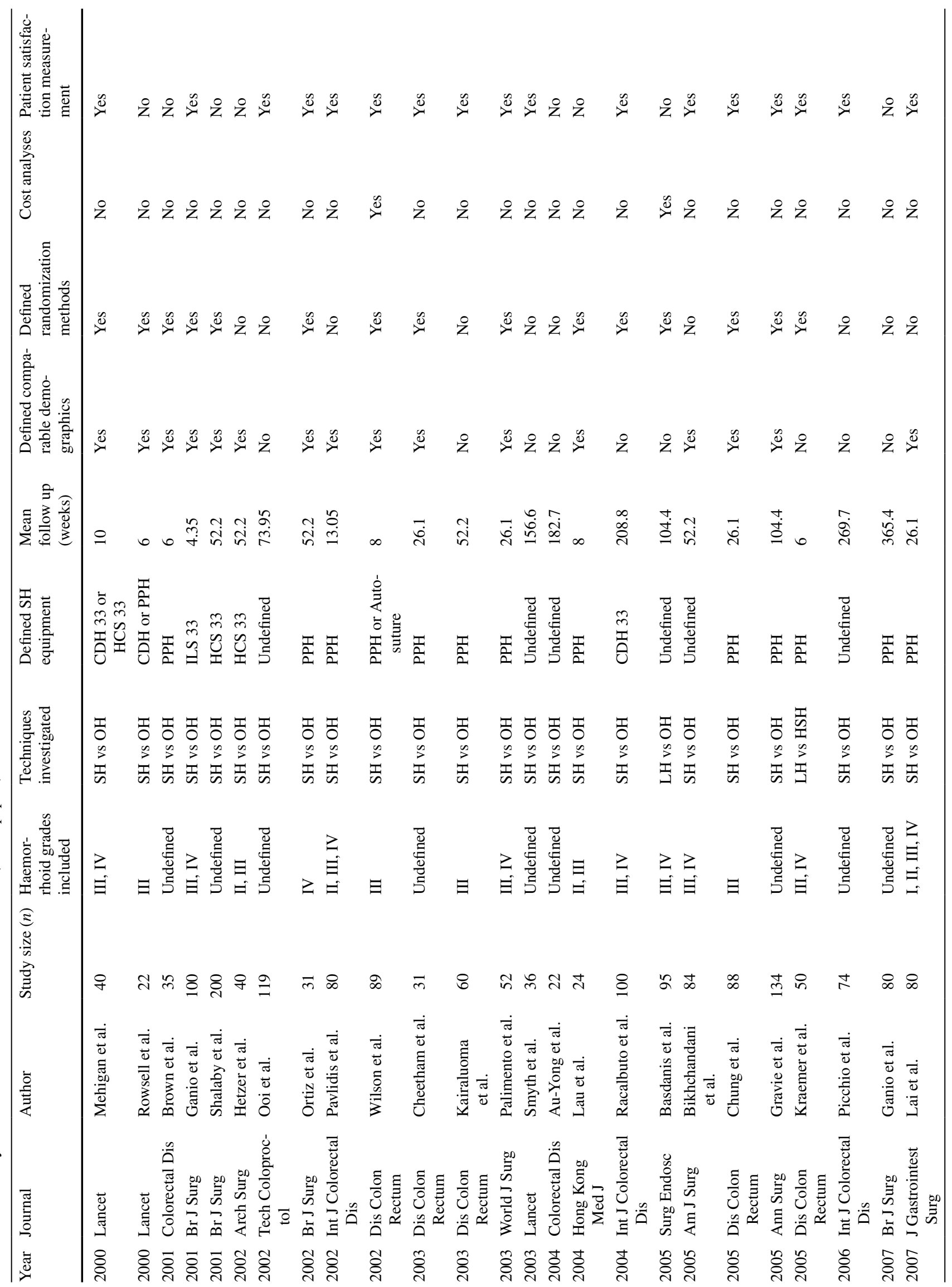




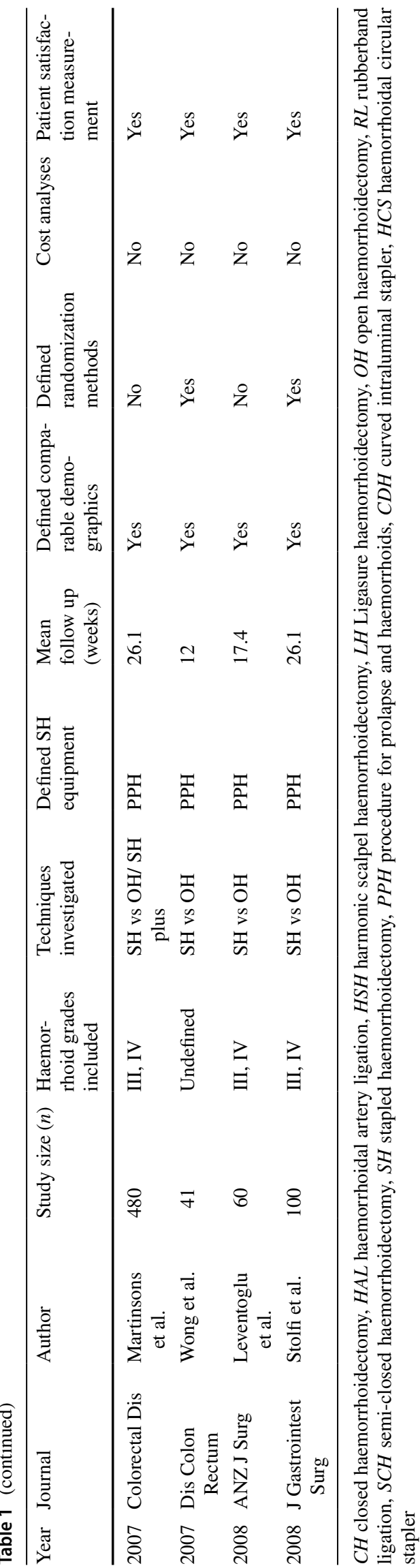

especially in the hours to days following surgery. More recent studies performed after 2008 explored pain control up to a year post procedure [47]. The advantages of SH in limiting short-term postoperative pain in this group largely corroborate with findings in the 1998-2008 group, while long-term benefits were more difficult to discern (Table 5).

\section{Long-term complications}

The long-term complications evaluated included fistulae, incontinence, anal stenosis, tenesmus, chronic pain and recurrence (Tables 6,7). There was limited evidence of any single complication being significantly more closely associated with SH compared to the other surgical approaches. Risks of incontinence and recurrence were the two complications most frequently measured by studies throughout the last 2 decades.

From 1998 to 2008, SH was associated with less chronic pain postoperatively, with two studies reaching significance. This was mirrored by Ripetti et al. [41] who were able to demonstrate a lower risk of anal stenosis $(p=0.004)$ and chronic rectal pain $(p<0.01)$ with SH in the 2009-2019 group. There was a study that suggested SH had a higher risk of causing postoperative tenesmus $(p=0.0012)$ [43]. As far as other complications were concerned, there were no differences between SH and OH. For recurrence, from 1998 to 2008 , close to half of the articles (5 of 14) raised concerns of higher recurrence in SH but only 2 studies reached significance, while from 2009 to 2019 , the number was reduced to 2 out of 7 with both studies reaching significance.

\section{Patient satisfaction}

From 1998 to 2008, 22 articles assessed postoperative patient satisfaction. Fourteen of the 22 articles failed to demonstrate a difference in satisfaction scores between $\mathrm{SH}$ and $\mathrm{OH}$ groups but 6 studies reported statistically significant satisfaction post SH (Table 8).

Regarding articles published from 2009 to 2019, Wang et al. compared overall satisfaction scores of $\mathrm{SH}$ and $\mathrm{OH}$, and reported a $97 \%$ satisfaction rate in $\mathrm{SH}$ vs $78 \%$ in $\mathrm{OH}$ [40]. An analysis of patient quality of life calculated via the EQ 5D 3L score was performed in the eTHOs trial by Watson et al. [43]. They demonstrated that scores were higher in the SH group up to 6 weeks postoperatively ( $p=0.0235)$. At 12 months, there was no statistically significant difference in satisfaction between $\mathrm{SH}$ and $\mathrm{OH}$ or closed haemorrhoidectomy $(\mathrm{CH})$ patient groups, but at 24 months, satisfaction became highest in the $\mathrm{OH} / \mathrm{CH}$ group $(p=0.0342)$. 


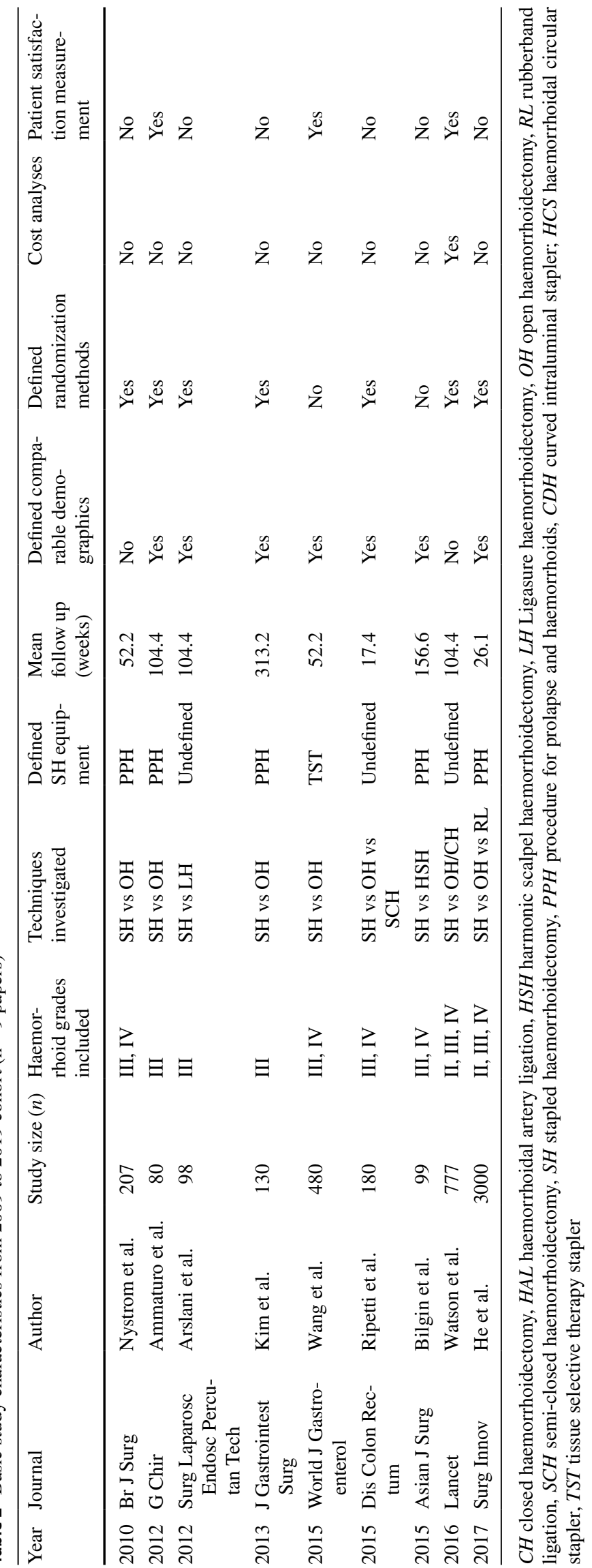


Table 3 Risks of shortterm complications (articles published from 1998 to 2008)

\begin{tabular}{|c|c|c|c|}
\hline Complication type & $\begin{array}{l}\text { Studies which assessed } \\
\text { defined complication }\end{array}$ & Study conclusions & $p$ value \\
\hline \multirow[t]{21}{*}{ Operative time } & Basdanis et al. & SH longer operative time vs $\mathrm{OH}$ & $<0.05$ \\
\hline & Brown et al. & SH longer operative time vs $\mathrm{OH}$ & $<0.05$ \\
\hline & Lau et al. & SH longer operative time vs $\mathrm{OH}$ & 0.26 \\
\hline & Chung et al. & SH shorter operative time vs HSH & 0.52 \\
\hline & Bikhchandani et al. & SH shorter operative time vs $\mathrm{OH}$ & $<0.001$ \\
\hline & Gravie et al. & SH shorter operative time vs $\mathrm{OH}$ & 0.035 \\
\hline & Hetzer et al. & SH shorter operative time vs $\mathrm{OH}$ & $<0.001$ \\
\hline & Kairaluoma et al. & $\mathrm{SH}$ shorter operative time vs $\mathrm{OH}$ & 0.49 \\
\hline & Kraemer et al. & SH shorter operative time vs $\mathrm{OH}$ & 0.1858 \\
\hline & Lai et al. & $\mathrm{SH}$ shorter operative time vs $\mathrm{OH}$ & $<0.01$ \\
\hline & Leventoglu et al. & SH shorter operative time vs $\mathrm{OH}$ & 0.0001 \\
\hline & Martinsons et al. & SH shorter operative time vs $\mathrm{OH}$ & 0.001 \\
\hline & Ortiz et al. & $\mathrm{SH}$ shorter operative time vs $\mathrm{OH}$ & 0.001 \\
\hline & Palimento et al. & $\mathrm{SH}$ shorter operative time vs $\mathrm{OH}$ & 0.041 \\
\hline & Pavlidis et al. & SH shorter operative time vs $\mathrm{OH}$ & $<0.05$ \\
\hline & Racalbuto et al. & SH shorter operative time vs $\mathrm{OH}$ & 0.164 \\
\hline & Shalaby et al. & SH shorter operative time vs $\mathrm{OH}$ & $<0.001$ \\
\hline & Wilson et al. & $\mathrm{SH}$ shorter operative time vs $\mathrm{OH}$ & $<0.001$ \\
\hline & Rowsell et al. & SH similar operative time vs $\mathrm{OH}$ & Undefined \\
\hline & Stolfi et al. & SH similar operative time vs $\mathrm{OH}$ & 0.94 \\
\hline & Wong et al. & SH similar operative time vs $\mathrm{OH}$ & 0.6 \\
\hline \multirow[t]{7}{*}{ Intraop blood loss } & Bikhchandani et al. & SH less blood loss vs $\mathrm{OH}$ & $<0.001$ \\
\hline & Chung et al. & SH less blood loss vs $\mathrm{OH}$ & 0.57 \\
\hline & Wilson et al. & SH less blood loss vs $\mathrm{OH}$ & $<0.001$ \\
\hline & Wong et al. & SH less blood loss vs $\mathrm{OH}$ & 0.58 \\
\hline & Basdanis et al. & SH more blood loss vs $\mathrm{OH}$ & $<0.05$ \\
\hline & Palimento et al. & SH more blood loss vs $\mathrm{OH}$ & 0.5 \\
\hline & Brown et al. & SH similar blood loss vs $\mathrm{OH}$ & Undefined \\
\hline \multirow[t]{18}{*}{ Postop bleeding } & Brown et al. & SH less blood loss vs $\mathrm{OH}$ & $<0.05$ \\
\hline & Cheetham et al. & SH less blood loss vs $\mathrm{OH}$ & 0.17 \\
\hline & Kraemer et al. & SH less blood loss vs $\mathrm{OH}$ & Undefined \\
\hline & Lai et al. & SH less blood loss vs $\mathrm{OH}$ & 1 \\
\hline & Leventoglu et al. & SH less blood loss vs $\mathrm{OH}$ & 0.017 \\
\hline & Ortiz et al. (2002) & SH less blood loss vs $\mathrm{OH}$ & Undefined \\
\hline & Shalaby et al. & SH less blood loss vs $\mathrm{OH}$ & Undefined \\
\hline & Stolfi et al. & SH less blood loss vs $\mathrm{OH}$ & $<0.001$ \\
\hline & Basdanis et al. & SH more blood loss vs $\mathrm{OH}$ & 0.5 \\
\hline & Gravie et al. & SH more blood loss vs $\mathrm{OH}$ & 0.477 \\
\hline & Hetzer et al. & SH more blood loss vs $\mathrm{OH}$ & Undefined \\
\hline & Kairaluoma et al. & $\mathrm{SH}$ more blood loss vs $\mathrm{OH}$ & Undefined \\
\hline & Palimento et al. & $\mathrm{SH}$ more blood loss vs $\mathrm{OH}$ & 1 \\
\hline & Pavlidis et al. & $\mathrm{SH}$ more blood loss vs $\mathrm{OH}$ & Undefined, ns \\
\hline & Racalbuto et al. & SH more blood loss vs $\mathrm{OH}$ & Undefined \\
\hline & Wilson et al. & SH more blood loss vs $\mathrm{OH}$ & Undefined \\
\hline & Ganio et al. & SH similar blood loss vs $\mathrm{OH}$ & Undefined, ns \\
\hline & Martinsons et al. & $\mathrm{SH}$ similar blood loss vs $\mathrm{OH}$ & 0.809 \\
\hline \multirow[t]{4}{*}{ Postop urinary retention } & Bikhchandani et al. & $\mathrm{SH}$ less retention vs $\mathrm{OH}$ & Undefined \\
\hline & Gravie et al. & $\mathrm{SH}$ less retention vs $\mathrm{OH}$ & 0.62 \\
\hline & Hetzer et al. & $\mathrm{SH}$ less retention vs $\mathrm{OH}$ & Undefined \\
\hline & Lau et al. & $\mathrm{SH}$ less retention vs $\mathrm{OH}$ & Undefined \\
\hline
\end{tabular}


Table 3 (continued)

\begin{tabular}{|c|c|c|c|}
\hline Complication type & $\begin{array}{l}\text { Studies which assessed } \\
\text { defined complication }\end{array}$ & Study conclusions & $p$ value \\
\hline & Leventoglu et al. & $\mathrm{SH}$ less retention vs $\mathrm{OH}$ & 0.017 \\
\hline & Ortiz et al. (2002) & $\mathrm{SH}$ less retention vs $\mathrm{OH}$ & Undefined \\
\hline & Palimento et al. & $\mathrm{SH}$ less retention vs $\mathrm{OH}$ & 0.54 \\
\hline & Racalbuto et al. & SH less retention vs $\mathrm{OH}$ & Undefined \\
\hline & Shalaby et al. & $\mathrm{SH}$ less retention vs $\mathrm{OH}$ & Undefined \\
\hline & Wong et al. & $\mathrm{SH}$ less retention vs $\mathrm{OH}$ & 0.48 \\
\hline & Chung et al. & $\mathrm{SH}$ more retention vs $\mathrm{HSH}$ & Undefined, ns \\
\hline & Basdanis et al. & $\mathrm{SH}$ more retention vs $\mathrm{OH}$ & $<0.5$ \\
\hline & Stolfi et al. & $\mathrm{SH}$ more retention vs $\mathrm{OH}$ & Undefined \\
\hline & Wilson et al. & $\mathrm{SH}$ more retention vs $\mathrm{OH}$ & Undefined \\
\hline & Lai et al. & $\mathrm{SH}$ similar retention vs $\mathrm{OH}$ & 1 \\
\hline & Martinsons et al. & $\mathrm{SH}$ similar retention vs $\mathrm{OH}$ & 0.243 \\
\hline & Mehigan et al. & $\mathrm{SH}$ similar retention vs $\mathrm{OH}$ & Undefined \\
\hline \multirow[t]{20}{*}{ Length of hospital stay } & Stolfi et al. & $\mathrm{SH}$ longer length of stay vs $\mathrm{OH}$ & 0.014 \\
\hline & Chung et al. & SH shorter length of stay vs HSH & 0.02 \\
\hline & Bikhchandani et al. & SH shorter length of stay vs $\mathrm{OH}$ & $<0.01$ \\
\hline & Ganio et al. & $\mathrm{SH}$ shorter length of stay vs $\mathrm{OH}$ & 0.01 \\
\hline & Gravie et al. & $\mathrm{SH}$ shorter length of stay vs $\mathrm{OH}$ & $<0.001$ \\
\hline & Hetzer et al. & SH shorter length of stay vs $\mathrm{OH}$ & 0.17 \\
\hline & Lai et al. & SH shorter length of stay vs $\mathrm{OH}$ & $<0.01$ \\
\hline & Lau et al. & $\mathrm{SH}$ shorter length of stay vs $\mathrm{OH}$ & 0.014 \\
\hline & Martinsions et al. & $\mathrm{SH}$ shorter length of stay vs $\mathrm{OH}$ & 0.001 \\
\hline & Pavlidis et al. & $\mathrm{SH}$ shorter length of stay vs $\mathrm{OH}$ & $<0.05$ \\
\hline & Racalbuto et al. & SH shorter length of stay vs $\mathrm{OH}$ & 0.098 \\
\hline & Rowsell et al. & SH shorter length of stay vs $\mathrm{OH}$ & $<0.001$ \\
\hline & Shalaby et al. & $\mathrm{SH}$ shorter length of stay vs $\mathrm{OH}$ & $<0.001$ \\
\hline & Wilson et al. & $\mathrm{SH}$ shorter length of stay vs $\mathrm{OH}$ & Undefined, ns \\
\hline & Wong et al. & $\mathrm{SH}$ shorter length of stay vs $\mathrm{OH}$ & 0.16 \\
\hline & Basdanis et al. & SH similar length of stay vs $\mathrm{OH}$ & Undefined \\
\hline & Brown et al. & SH similar length of stay vs $\mathrm{OH}$ & Undefined \\
\hline & Kairaluoma et al. & $\mathrm{SH}$ similar length of stay vs $\mathrm{OH}$ & 0.1 \\
\hline & Kraemer et al. & $\mathrm{SH}$ similar length of stay vs $\mathrm{OH}$ & Undefined \\
\hline & Mehigan et al. & $\mathrm{SH}$ similar length of stay vs $\mathrm{OH}$ & 0.05 \\
\hline
\end{tabular}

$\mathrm{OH}$ open haemorrhoidectomy, $S H$ stapled haemorrhoidectomy, $H S H$ harmonic scalpel haemorrhoidectomy

\section{Cost}

The cost of treatments was mentioned in three of the studies. In 2002, Wilson et al. calculated that open haemorrhoidectomy cost $\$ 1798$ per procedure, higher than that of Autosuture stapled anopexy (\$1156) and Ethicon stapled anopexy (\$1312). In 2016, the eTHOs trial showed that SH cost $£ 941$ per patient and $\mathrm{OH}$ or $\mathrm{CH}$ cost $£ 602$ per patient, concluding that $\mathrm{SH}$ costs more and provides a lower number of qualityadjusted life years (QALYs) per patient than $\mathrm{OH}$ or $\mathrm{CH}$ [43]. Bilgin et al. [42] did not perform a formal cost analysis but pointed out that the equipment costs for the harmonic scalpel were double that for $\mathrm{SH}$.

\section{Discussion}

SH was introduced in 1998 as a conceptually attractive surgical technique as it mobilizes the prolapsed rectal mucosa above the dentate line, back to its original anatomical position [48]. Since stapling is meant to be performed above the dentate line on insensate rectal mucosa, postoperative pain and discomfort ought to be minimized, thereby positively influencing length of stay [42]. Resection of a circumferential ring of rectal mucosa eliminates all distal feeding vessels from the superior rectal artery, theoretically attaining a higher degree of surgical completeness and an expected lower risk of recurrence. 
Table 4 Risks of short-term complications in articles published from 2009 to 2019

\begin{tabular}{|c|c|c|c|}
\hline Complication type & $\begin{array}{l}\text { Studies which assessed } \\
\text { defined complication }\end{array}$ & Study conclusions & $p$ value \\
\hline \multirow[t]{8}{*}{ Operative time } & He et al. & SH shorter intraop time vs $\mathrm{OH}$ & $<0.05$ \\
\hline & Kim et al. & SH shorter intraop time vs $\mathrm{OH}$ & $<0.001$ \\
\hline & Ripetti et al. & SH shorter intraop time vs $\mathrm{OH}$ & Undefined \\
\hline & Wang et al. & SH shorter intraop time vs $\mathrm{OH}$ & $<0.001$ \\
\hline & Ammaturo et al. & SH shorter intraop time vs $\mathrm{OH}$ & Undefined \\
\hline & Nystrom et al. & SH similar intraop time vs $\mathrm{OH}$ & 0.247 \\
\hline & Watson et al. & SH similar intraop time vs THD & Undefined \\
\hline & Bilgin et al. & SH longer intraop time vs HSH & Undefined \\
\hline Intraop blood loss & Wang et al. & SH less blood loss vs $\mathrm{OH}$ & $<0.001$ \\
\hline Postop burning sensation & Kim et al. & SH less symptomatic than $\mathrm{OH}$ & $<0.001$ \\
\hline \multirow[t]{8}{*}{ Postop bleeding } & Ammaturo et al. & SH less blood loss vs $\mathrm{OH}$ & Undefined \\
\hline & Nystrom et al. & SH less blood loss vs $\mathrm{OH}$ & Undefined \\
\hline & Arslani et al. & SH similar blood loss vs $\mathrm{OH}$ & 0.504 \\
\hline & Kim et al. & SH similar blood loss vs $\mathrm{OH}$ & Undefined \\
\hline & Ripetti et al. & SH similar blood loss vs $\mathrm{OH} / \mathrm{SCH}$ & 0.21 \\
\hline & Bilgin et al. & SH more blood loss vs HSH & Undefined \\
\hline & He et al. & SH more blood loss vs $\mathrm{OH}$ & $<0.05$ \\
\hline & Watson et al. & SH more blood loss vs $\mathrm{OH} / \mathrm{CH}$ & Undefined \\
\hline \multirow[t]{6}{*}{ Postop urinary retention } & Ammaturo et al. & $\mathrm{SH}$ less retention risk vs $\mathrm{OH}$ & Undefined \\
\hline & He et al. & $\mathrm{SH}$ less retention risk vs $\mathrm{OH}$ & $<0.05$ \\
\hline & Wang et al. & $\mathrm{SH}$ less retention risk vs $\mathrm{OH}$ & 0.001 \\
\hline & Arslani et al. & $\mathrm{SH}$ similar retention risk vs $\mathrm{OH}$ & 0.898 \\
\hline & Kim et al. & $\mathrm{SH}$ similar retention risk vs $\mathrm{OH}$ & 1 \\
\hline & Ripetti et al. & $\mathrm{SH}$ similar retention risk vs $\mathrm{OH}, \mathrm{SCH}$ & 0.2 \\
\hline \multirow[t]{6}{*}{ Length of hospital stay } & Ammaturo et al. & $\mathrm{SH}$ shorter length of stay vs $\mathrm{OH}$ & Undefined \\
\hline & He et al. & $\mathrm{SH}$ shorter length of stay vs $\mathrm{OH}$ & $<0.05$ \\
\hline & Wang et al. & SH shorter length of stay vs $\mathrm{OH}$ & $<0.01$ \\
\hline & Bilgin et al. & SH similar length of stay vs HSH & Undefined \\
\hline & Nystrom et al. & SH similar length of stay vs $\mathrm{OH}$ & 0.456 \\
\hline & Watson et al. & $\mathrm{SH}$ similar length of stay vs $\mathrm{OH} / \mathrm{CH}$ & Undefined \\
\hline \multirow[t]{2}{*}{ Wound infection } & Ammaturo et al. & SH similar infection risk vs $\mathrm{OH}$ & Undefined \\
\hline & Watson et al. & $\mathrm{SH}$ similar infection risk vs $\mathrm{OH} / \mathrm{CH}$ & Undefined \\
\hline
\end{tabular}

$\mathrm{CH}$ closed haemorrhoidectomy, $\mathrm{HSH}$ harmonic scalpel haemorrhoidectomy, $\mathrm{OH}$ open haemorrhoidectomy, SCH semi-closed haemorrhoidectomy, SH stapled haemorrhoidectomy, THD transanal haemorrhoidal dearterialization
This systematic review appears to show that $\mathrm{SH}$ is a safe procedure potentially associated with decreased intraoperative blood loss and operative times. It is interesting to note that the shorter SH operative time was already well demonstrated early on (1998-2008) but did not improve further during the latter time period (2009-2019), as shown in Tables 3, 4. In fact, the operative time did not change across the two decades, perhaps indicating that a plateau is reached early beyond which further improvement is not possible [20].

SH seems to be less painful in the immediate postoperative period leading to less urinary retention. In the longer term, although pain was less frequent post $\mathrm{SH}$, overall patient satisfaction appears to decline with time with $\mathrm{OH} /$
$\mathrm{CH}$ associated with greater quality of life scores after 2 years [43]. Despite the lack of formal statistical analysis when it came to cost-evaluation, mainly due to the fact that cost was not consistently reported in all studies, $\mathrm{SH}$ is probably less cost efficient compared to $\mathrm{OH}$ or $\mathrm{CH}$, although the supporters of the procedure state that it is not the most resourcedemanding procedure on the market and savings are accrued through reduced operative time and shorter length of hospital stay. However, the eTHos trial reported a lower number of QALYs casting further doubts on its potential advantages [43].

Recurrence is an important measure of technical efficacy and $\mathrm{SH}$ appears to be just as effective as other surgical interventions. Reports in the past had shown concerns about 
Table 5 Visual analogue scale for pain as demonstrated by individual studies

\begin{tabular}{|c|c|c|c|c|}
\hline \multicolumn{5}{|c|}{ Visual analogue scale (VAS) } \\
\hline Author & $\begin{array}{l}\text { Postoperative duration at } \\
\text { pain measurement }\end{array}$ & SH & $\begin{array}{l}\mathrm{CH} / \mathrm{HAL} / \mathrm{HSH} / \\
\mathrm{OH} / \mathrm{SCH} / \mathrm{SH}\end{array}$ & $p$ value \\
\hline \multicolumn{5}{|l|}{ 1998-2008 } \\
\hline \multirow[t]{2}{*}{ Basdanis et al. } & 1 day & 5 & 7 & $<0.001$ \\
\hline & 1 week & 1 & 2 & Undefined \\
\hline \multirow[t]{5}{*}{ Bikhchandani et al. } & $12 \mathrm{~h}$ & 3.45 & 4.86 & $<0.001$ \\
\hline & 1 day & 3.64 & 6.36 & $<0.001$ \\
\hline & 3 days & 1.52 & 4.5 & $<0.001$ \\
\hline & 1 week & 0.57 & 2.31 & $<0.01$ \\
\hline & 15 days & 0.21 & 1.05 & $<0.001$ \\
\hline Cheetham et al. & 10 days & 4.5 & 9 & 0.018 \\
\hline Chung et al. & 7 days & 1.5 & 3.5 & 0.002 \\
\hline Gravie et al. & 10 days & Undefined & Undefined & $<0.001$ (in favour of $\mathrm{SH}$ ) \\
\hline \multirow[t]{4}{*}{ Hetzer et al. } & 1 day & 2.7 & 6.3 & $<0.01$ \\
\hline & 2 days & 1.7 & 6.3 & $<0.01$ \\
\hline & 3 days & 0.8 & 5.4 & $<0.01$ \\
\hline & 4 days & 0.5 & 4.8 & $<0.01$ \\
\hline Kraemer et al. & 3 weeks & Undefined & Undefined & 0.99 \\
\hline \multirow[t]{3}{*}{ Lai et al. } & 1 day & 3.53 & 7.18 & $<0.01$ \\
\hline & 7 days & 1.98 & 3.68 & $<0.01$ \\
\hline & 2 weeks & 1.33 & 1.85 & $<0.01$ \\
\hline Lau et al. & 2 days & 4 & 3.1 & 0.93 \\
\hline \multirow[t]{6}{*}{ Leventoglu et al. } & $8 \mathrm{~h}$ & 5.7 & 7.75 & 0.0001 \\
\hline & 1 day & 1.3 & 4.5 & 0.0001 \\
\hline & 2 days & 0.9 & 3 & 0.025 \\
\hline & 7 days & 0.15 & 1.5 & 0.026 \\
\hline & 2 weeks & 0 & 1 & 0.014 \\
\hline & 4 weeks & 0 & 1 & 0.015 \\
\hline Palimento et al. & $4 \mathrm{~h}$ & Undefined & Undefined & $<0.001$ (in favour of $\mathrm{SH}$ ) \\
\hline \multirow[t]{4}{*}{ Pavlidis et al. } & $3 \mathrm{~h}$ & 2.5 & 3.4 & $<0.05$ \\
\hline & $6 \mathrm{~h}$ & 2.9 & 3.9 & $<0.05$ \\
\hline & $12 \mathrm{~h}$ & 2.3 & 3.6 & $<0.05$ \\
\hline & 1 day & 0.7 & 2.4 & $<0.01$ \\
\hline \multirow[t]{2}{*}{ Shalaby et al. } & 1 day & 2.5 & 7.6 & $<0.001$ \\
\hline & 1 week & 0.4 & 2.6 & $<0.001$ \\
\hline \multirow[t]{2}{*}{ Stolfi et al. } & 2 days & 5.11 & 5.13 & 0.96 \\
\hline & 8 days & 3.98 & 4.82 & 0.016 \\
\hline Wong et al. & 1 week & 4.1 & 5.7 & 0.02 \\
\hline \multicolumn{5}{|l|}{ 2009-2019 } \\
\hline \multirow[t]{3}{*}{ Kim et al. } & 1 week & 3.1 & 6.2 & $<0.001$ \\
\hline & 2 weeks & 0.5 & 3 & $<0.001$ \\
\hline & 4 weeks & 0.05 & 0.6 & $<0.001$ \\
\hline Lehur et al. & 2 weeks & 2.8 & 2.2 & 0.03 \\
\hline \multirow[t]{4}{*}{ Leung et al. } & 1 week & 3.7 & 3.4 & 0.09 \\
\hline & 2 months & 1 & 1 & 0.2 \\
\hline & 4 months & 1 & 1 & 0.079 \\
\hline & 1 year & 1 & 1 & 0.767 \\
\hline Wang et al. & $12 \mathrm{~h}$ & 5.1 & 7.2 & $<0.001$ \\
\hline \multirow[t]{3}{*}{ Watson et al. } & 1 week & 4 & 5.3 & $<0.0001$ \\
\hline & 3 weeks & 1.8 & 2.6 & 0.0026 \\
\hline & 6 weeks & 1.3 & 1.3 & 0.96 \\
\hline
\end{tabular}

$\mathrm{CH}$ closed haemorrhoidectomy, $H A L$ haemorrhoidal artery ligation, $H S H$ harmonic scalpel haemorrhoidectomy, $\mathrm{OH}$ open haemorrhoidectomy, $\mathrm{SCH}$ semi-closed haemorrhoidectomy, $\mathrm{SH}$ stapled haemorrhoidectomy, THD transanal haemorrhoidal dearterialization 
Table 6 Risks of long-term complications in articles published in 1998-2008

\begin{tabular}{|c|c|c|c|}
\hline Complication type & $\begin{array}{l}\text { Studies which assessed } \\
\text { defined complication }\end{array}$ & Study conclusions & $p$ value \\
\hline Fistulae & Ortiz et al. & $\mathrm{SH}$ lower risk of fistula formation vs $\mathrm{OH}$ & Undefined \\
\hline \multirow[t]{11}{*}{ Incontinence } & Bikhchandani et al. & SH lower risk of incontinence vs $\mathrm{OH}$ & Undefined \\
\hline & Mehigan et al. & $\mathrm{SH}$ lower risk of incontinence vs $\mathrm{OH}$ & Undefined \\
\hline & Pavlidis et al. & $\mathrm{SH}$ lower risk of incontinence vs $\mathrm{OH}$ & Undefined \\
\hline & $\mathrm{Au}$ Yong et al. & SH similar risk of incontinence vs $\mathrm{OH}$ & 0.56 \\
\hline & Ganio et al. & $\mathrm{SH}$ similar risk of incontinence vs $\mathrm{OH}$ & 0.479 \\
\hline & Gravie et al. & $\mathrm{SH}$ similar risk of incontinence vs $\mathrm{OH}$ & 0.29 \\
\hline & Hetzer et al. & $\mathrm{SH}$ similar risk of incontinence vs $\mathrm{OH}$ & Undefined \\
\hline & Kairaluoma et al. & SH similar risk of incontinence vs $\mathrm{OH}$ & 0.61 \\
\hline & Kraemer et al. & $\mathrm{SH}$ similar risk of incontinence vs $\mathrm{OH}$ & Undefined \\
\hline & Leventoglu et al. & SH similar risk of incontinence vs $\mathrm{OH}$ & 0.114 \\
\hline & Smyth et al. & SH similar risk of incontinence vs $\mathrm{OH}$ & 0.409 \\
\hline \multirow[t]{7}{*}{ Anal stenosis } & $\mathrm{Au}$ Yong et al. & SH similar risk of stenosis vs $\mathrm{OH}$ & Undefined \\
\hline & Bikhchandani et al. & $\mathrm{SH}$ similar risk of stenosis vs $\mathrm{OH}$ & Undefined \\
\hline & Brown et al. & $\mathrm{SH}$ similar risk of stenosis vs $\mathrm{OH}$ & ns \\
\hline & Ganio et al. & $\mathrm{SH}$ similar risk of stenosis vs $\mathrm{OH}$ & Undefined \\
\hline & Gravie et al. & SH similar risk of stenosis vs $\mathrm{OH}$ & 1 \\
\hline & Martinsons et al. & SH similar risk of stenosis vs $\mathrm{OH}$ & 0.663 \\
\hline & Shalaby et al. & SH similar risk of stenosis vs $\mathrm{OH}$ & Undefined \\
\hline \multirow[t]{7}{*}{ Chronic pain } & Bikhchandani et al. & SH lower risk of pain vs $\mathrm{OH}$ & Undefined \\
\hline & Brown et al. & $\mathrm{SH}$ lower risk of pain vs $\mathrm{OH}$ & $<0.05$ \\
\hline & Kraemer et al. & $\mathrm{SH}$ lower risk of pain vs $\mathrm{OH}$ & Undefined \\
\hline & Martinsons et al. & $\mathrm{SH}$ lower risk of pain vs $\mathrm{OH}$ & $<0.001$ \\
\hline & Kairaluoma et al. & SH similar risk of pain vs $\mathrm{OH}$ & 1 \\
\hline & Ooi et al. & SH similar risk of pain vs $\mathrm{OH}$ & Undefined \\
\hline & Picchio et al. & $\mathrm{SH}$ similar risk of pain vs $\mathrm{OH}$ & 1 \\
\hline \multirow[t]{7}{*}{ Tenesmus/ Urgency } & Ganio et al. & SH higher risk of urgency vs $\mathrm{OH}$ & Undefined \\
\hline & Gravie et al. & $\mathrm{SH}$ similar risk of tenesmus vs $\mathrm{OH}$ & 1 \\
\hline & Au Yong et al. & SH similar risk of urgency vs $\mathrm{OH}$ & 0.41 \\
\hline & Mehigan et al. & $\mathrm{SH}$ similar risk of urgency vs $\mathrm{OH}$ & Undefined \\
\hline & Ortiz et al. & SH similar risk of urgency vs $\mathrm{OH}$ & Undefined \\
\hline & Smyth et al. & $\mathrm{SH}$ similar risk of urgency vs $\mathrm{OH}$ & undefined \\
\hline & Stolfi et al. & SH similar risk of urgency vs $\mathrm{OH}$ & Undefined \\
\hline \multirow[t]{14}{*}{ Recurrence } & Basdanis et al. & $\mathrm{SH}$ higher risk of recurrence vs $\mathrm{OH}$ & Undefined \\
\hline & Bikhchandani et al. & $\mathrm{SH}$ higher risk of recurrence vs $\mathrm{OH}$ & Undefined \\
\hline & Ganio et al. & $\mathrm{SH}$ higher risk of recurrence vs $\mathrm{OH}$ & 0.001 \\
\hline & Ortiz et al. & $\mathrm{SH}$ higher risk of recurrence vs $\mathrm{OH}$ & 0.004 \\
\hline & Racalbuto & $\mathrm{SH}$ higher risk of recurrence vs $\mathrm{OH}$ & Undefined \\
\hline & Wong et al. & $\mathrm{SH}$ lower risk of recurrence vs $\mathrm{OH}$ & 0.002 \\
\hline & Chung et al. & $\mathrm{SH}$ similar risk of recurrence vs $\mathrm{HSH}$ & 0.93 \\
\hline & Au Yong et al. & $\mathrm{SH}$ similar risk of recurrence vs $\mathrm{OH}$ & 0.57 \\
\hline & Cheetham et al. & $\mathrm{SH}$ similar risk of recurrence vs $\mathrm{OH}$ & ns \\
\hline & Ganio et al. & $\mathrm{SH}$ similar risk of recurrence vs $\mathrm{OH}$ & 0.562 \\
\hline & Gravie et al. & $\mathrm{SH}$ similar risk of recurrence vs $\mathrm{OH}$ & 0.498 \\
\hline & Ooi et al. & $\mathrm{SH}$ similar risk of recurrence vs $\mathrm{OH}$ & ns \\
\hline & Shalaby et al. & $\mathrm{SH}$ similar risk of recurrence vs $\mathrm{OH}$ & Undefined \\
\hline & Stolfi et al. & $\mathrm{SH}$ similar risk of recurrence vs $\mathrm{OH}$ & 0.17 \\
\hline
\end{tabular}

$H S H$ harmonic scalpel haemorrhoidectomy, $O H$ open haemorrhoidectomy, $S H$ stapled haemorrhoidectomy, $n s$ not significant 
Table 7 Risks of long-term complications in articles published 2009-2019

\begin{tabular}{llll}
\hline Complication type & $\begin{array}{l}\text { Studies which assessed } \\
\text { defined complication }\end{array}$ & Study conclusions & $p$ value \\
\hline Fistulae & Kim et al. & SH lower risk of fistula formation vs OH & $\mathrm{ns}$ \\
& Ripetti et al. & SH similar risk of fistula formation vs OH/ SCH & 0.39 \\
& Bilgin et al. & SH higher risk of fistula formation vs HSH & $\mathrm{ns}$ \\
Incontinence & Wang et al. & SH lower risk of incontinence vs OH & $<0.05$ \\
& Kim et al. & SH similar risk of incontinence vs OH & 0.559 \\
& Lehur et al. & SH similar risk of incontinence vs HAL & $\mathrm{ns}$ \\
& Ripetti et al. & SH similar risk of incontinence vs OH/ SCH & 0.38 \\
& Watson et al. & SH higher risk of incontinence vs OH/ CH & $\mathrm{ns}$ \\
Anal stenosis & Bilgin et al. & SH lower risk of stenosis vs HSH & $\mathrm{ns}$ \\
& Ripetti et al. & SH lower risk of stenosis vs OH & 0.004 \\
& Watson et al. & SH higher risk of stenosis vs OH & $\mathrm{ns}$ \\
Chronic pain & Ripetti et al. & SH lower risk of chronic pain vs OH/ SCH & $<0.01$ \\
& Lehur et al. & SH similar risk of chronic pain vs HAL & 0.87 \\
Tenesmus/urgency & Watson et al. & SH higher risk of tenesmus vs OH/ CH & 0.0012 \\
Recurrence & Giarratano et al. & SH lower risk of recurrence vs THD & 0.04 \\
& Leung et al. & SH lower risk of recurrence vs THD & $<0.00001$ \\
& Kim et al. & SH similar risk of recurrence vs OH & 0.65 \\
& Lehur et al. & SH similar risk of recurrence vs HAL & 0.65 \\
& Ripetti et al. & SH similar risk of recurrence vs OH/SCH & 0.8 \\
& Bilgin et al. & SH higher risk of recurrence vs HSH & $<0.05$ \\
& Watson et al. & SH higher risk of recurrence vs OH & $<0.0001$ \\
\hline
\end{tabular}

$\mathrm{CH}$ closed haemorrhoidectomy, $H A L$ haemorrhoidal artery ligation, $H S H$ harmonic scalpel haemorrhoidectomy, $\mathrm{OH}$ open haemorrhoidectomy, $\mathrm{SCH}$ semi-closed haemorrhoidectomy, $\mathrm{SH}$ stapled haemorrhoidectomy, THD transanal haemorrhoidal dearterialization, $n s$ not significant using SH on grade IV haemorrhoids due to the risk of higher recurrence but this has not been convincingly demonstrated. In our review, the risk of recurrence was deemed similar in $\mathrm{SH}$ and $\mathrm{OH}$ through both decades, with a smaller fraction of published articles demonstrating higher recurrence risks in SH from 2009 to 2019 compared to 1998-2008, possibly due to increased experience and improved stapling devices. Doppler-guided transanal haemorrhoidal dearterialization (THD) did, however, seem to consistently be associated with higher recurrence rates than $\mathrm{SH}$ [45]. Furthermore, despite the inability of SH to excise external haemorrhoidal components, it was often observed that the external lesions shrink due to disruption of their blood supply [34]. It has also been suggested that as the stapling preserves the haemorrhoid tissues and seeks only to disrupt its blood supply, its elimination is more physiological than outright excision, therefore reducing the risk of anal stenosis [12], which supports the findings in Table 7.

Severe complications such as deep pelvic sepsis and peritonitis are recognised but rare complications of haemorrhoidal surgery. It was thought that $\mathrm{SH}$, due to the extent of its tissue manipulation and circumferential involvement of the rectal mucosa, would dispose patients to a higher risk of deep infections. Furthermore, rectal perforation was considered to be an almost exclusive complication of $\mathrm{SH}$ from inappropriate deployment of the stapling mechanism [52]. Our review did not identify a single case of these complications although anecdotally they have occurred. Furthermore, it is worthy of note that several other high-volume techniques used in haemorrhoid surgery such as Doppler-guided THD [53] as well as haemorrhoid laser procedure (HeLP) [54] have been associated with postoperative complications necessitating faecal diversion. These occurrences show that the potential for postoperative morbidity is not exclusive to $\mathrm{SH}$. Nonetheless, one must be vigilant in recognizing rare complications such as rectal pocket syndrome [55], rectocoele and rectal intussusception [56] so as to prevent severe pelvic or intra-abdominal sepsis.

Studies were keen to include patients' self-rated satisfaction levels following surgery in an attempt to quantify the more subjective components of a successful procedure. The most comprehensive of them used the EQ 5D 3L, which captures personal dimensions of mobility, self-care, activity, pain, anxiety as well as an overall self-rated well-being score [57]. SH was good in measurements of patient satisfaction in most selected RCTs we evaluated [40, 47] even when placed under the scrutiny of EQ 5DL 3L, although satisfaction appeared to decline with time [43].

Current evidence suggests that $\mathrm{SH}$ is a safe surgical option in the management of haemorrhoid disease. It 
Table 8 Articles with patient expressed satisfaction or quality of life scoring post haemorrhoidectomy

\begin{tabular}{lll}
\hline Author & $p$ value & Article conclusion \\
\hline 1998-2008 & & \\
Basdanies et al. & Undefined & SH similar to OH \\
Cheetham et al. & 0.76 & SH similar to OH \\
Gravie et al. & Undefined & SH similar to OH \\
Kairaluoma et al. & 0.2 & SH similar to OH \\
Kraemer et al. & 1 & SH similar to OH \\
Lai et al. & 0.39 & SH similar to OH \\
Mehigan et al. & Undefined & SH similar to OH \\
Ooi et al. & Undefined & SH similar to OH \\
Picchio et al. & undefined & SH similar to OH \\
Smyth et al. & Undefined & SH similar to OH \\
Wilson et al.* & Undefined & SH similar to OH \\
Ganio et al. & 0.33 & SH similar to OH \\
Palimento et al. & 0.735 & SH similar to OH \\
Stolfi et al. & Undefined & SH similar to OH \\
Bikhchandani et al. & $<0.01$ & SH superior \\
Chunget al. & 0.001 & SH superior \\
Leventoglu et al. & 0.008 & SH superior \\
Wong et al. & 0.03 & SH superior \\
Pavlidis et al. & $<0.05$ & SH superior \\
Racalbuto & $<0.0001$ & SH superior \\
Martinsons et al.* & Undefined & Undefined \\
Ortiz et al. & Undefined & Undefined \\
2009-2019 & & \\
Ammaturo et al. & Undefined & SH similar to OH \\
Watson et al.* & Undefined & SH similar to OH \\
Wang et al. & $<0.01$ & SH superior \\
\hline & & \\
\hline
\end{tabular}

$\mathrm{OH}$ open haemorrhoidectomy, $\mathrm{SH}$ stapled haemorrhoidectomy

*Studies with quality of life assessment without satisfaction scoring

continues to be embraced by many in the field as a robust and reliable technique with potential, but not fully proven, advantages, at least in the short term. Nevertheless, it has not been found to be inferior to other techniques in this review. The concerns regarding the use of staples remain, which explains why the technique has not been widely adopted in the UK and many other countries. Perhaps the use of biodegradable staples may alleviate some of these concerns and further improve its safety profile.

\section{Conclusions}

The evidence is lacking at the moment to suggest routine use of SH but it can be safely considered in selected patients. It is still not known what group of patients is most likely to benefit from the procedure.
Author contributions All authors contributed to the literature review and the preparation of this manuscript.

\section{Compliance with ethical standards}

Conflict of interest The authors declare that they have no conflict of interest.

Ethical approval This article does not contain any studies with human participants or animals performed by any of the authors.

Informed consent For this type of study formal consent is not required.

Open Access This article is licensed under a Creative Commons Attribution 4.0 International License, which permits use, sharing, adaptation, distribution and reproduction in any medium or format, as long as you give appropriate credit to the original author(s) and the source, provide a link to the Creative Commons licence, and indicate if changes were made. The images or other third party material in this article are included in the article's Creative Commons licence, unless indicated otherwise in a credit line to the material. If material is not included in the article's Creative Commons licence and your intended use is not permitted by statutory regulation or exceeds the permitted use, you will need to obtain permission directly from the copyright holder. To view a copy of this licence, visit http://creativecommons.org/licenses/by/4.0/.

\section{References}

1. Sun Z, Migaly J (2016) Review of hemorrhoid disease: presentation and management. Clin Colon Rectal Surg 29(1):22-29

2. Loder PB, Kamm MA, Nicholls RJ, Phillips RK (1994) Haemorrhoids: pathology, pathophysiology and aetiology. Br J Surg 81(7):946-954

3. Hardy A, Chan CL, Cohen CR (2005) The surgical management of haemorrhoids - a review. Dig Surg 22(1-2):26-33

4. Jayaraman S, Colquhoun PH, Malthaner RA (2006) Stapled versus conventional surgery for hemorrhoids. Cochrane Database Syst Rev (4):CD005393. https://doi.org/10.1002/14651858.CD005393. pub2

5. Ho YH, Seow-Choen F, Tsang C, Eu KW (2001) Randomized trial assessing anal sphincter injuries after stapled haemorrhoidectomy. Br J Surg 88(11):1449-55. https://doi.org/10.104 6/j.0007-1323.2001.01899.x

6. Liberati A, Altman DG, Tetzlaff J, Mulrow C, Gotzsche PC, Ioannidis JP et al (2009) The PRISMA statement for reporting systematic reviews and meta-analyses of studies that evaluate healthcare interventions: explanation and elaboration. BMJ 339:b2700

7. Mehigan BJ, Monson JR, Hartley JE (2000) Stapling procedure for haemorrhoids versus Milligan-Morgan haemorrhoidectomy: randomised controlled trial. Lancet 355(9206):782-785. https:// doi.org/10.1016/S0140-6736(99)08362-2

8. Rowsell M, Bello M, Hemingway DM (2000) Circumferential mucosectomy (stapled haemorrhoidectomy) versus conventional haemorrhoidectomy: randomised controlled trial. Lancet 355(9206):779-781

9. Hetzer FH, Demartines N, Handschin AE, Clavien PA (2002) Stapled vs excision hemorrhoidectomy: long-term results of a prospective randomized trial. Arch Surg 137(3):337-340

10. Shalaby R, Desoky A (2001) Randomized clinical trial of stapled versus Milligan-Morgan haemorrhoidectomy. Br J Surg 88(8):1049-1053 
11. Smyth EF, Baker RP, Wilken BJ, Hartley JE, White TJ, Monson JR (2003) Stapled versus excision haemorrhoidectomy: long-term follow up of a randomised controlled trial. Lancet 361(9367):1437-1438

12. Bikhchandani J, Agarwal PN, Kant R, Malik VK (2005) Randomized controlled trial to compare the early and mid-term results of stapled versus open hemorrhoidectomy. Am J Surg 189(1):56-60

13. Ganio E, Altomare DF, Gabrielli F, Milito G, Canuti S (2001) Prospective randomized multicentre trial comparing stapled with open haemorrhoidectomy. Br J Surg 88(5):669-674

14. Pavlidis T, Papaziogas B, Souparis A, Patsas A, Koutelidakis I, Papaziogas T (2002) Modern stapled Longo procedure vs conventional Milligan-Morgan hemorrhoidectomy: a randomized controlled trial. Int J Colorectal Dis. 17(1):50-53

15. Ooi BS, Ho YH, Tang CL, Eu KW, Seow-Choen F (2002) Results of stapling and conventional hemorrhoidectomy. Tech Coloproctol. 6(1):59-60 (author reply 1-2)

16. Ortiz H, Marzo J, Armendariz P (2002) Randomized clinical trial of stapled haemorrhoidopexy versus conventional diathermy haemorrhoidectomy. Br J Surg 89(11):1376-1381

17. Wilson MS, Pope V, Doran HE, Fearn SJ, Brough WA (2002) Objective comparison of stapled anopexy and open hemorrhoidectomy: a randomized, controlled trial. Dis Colon Rectum 45(11):1437-1444

18. Kairaluoma M, Nuorva K, Kellokumpu I (2003) Day-case stapled (circular) vs. diathermy hemorrhoidectomy: a randomized, controlled trial evaluating surgical and functional outcome. Dis Colon Rectum. 46(1):93-99

19. Palimento D, Picchio M, Attanasio U, Lombardi A, Bambini C, Renda A (2003) Stapled and open hemorrhoidectomy: randomized controlled trial of early results. World J Surg 27(2):203-207

20. Cheetham MJ, Cohen CR, Kamm MA, Phillips RK (2003) A randomized, controlled trial of diathermy hemorrhoidectomy vs. stapled hemorrhoidectomy in an intended day-care setting with longer-term follow-up. Dis Colon Rectum. 46(4):491-497

21. Brown SR, Ballan K, Ho E, Ho Fams YH, Seow-Choen F (2001) Stapled mucosectomy for acute thrombosed circumferentially prolapsed piles: a prospective randomized comparison with conventional haemorrhoidectomy. Colorectal Dis 3(3):175-178

22. Racalbuto A, Aliotta I, Corsaro G, Lanteri R, Di Cataldo A, Licata A (2004) Hemorrhoidal stapler prolapsectomy vs. Milligan-Morgan hemorrhoidectomy: a long-term randomized trial. Int J Colorectal Dis. 19(3):239-244

23. Au-Yong I, Rowsell M, Hemingway DM (2004) Randomised controlled clinical trial of stapled haemorrhoidectomy vs conventional haemorrhoidectomy; a three and a half year follow up. Colorectal Dis 6(1):37-38

24. Basdanis G, Papadopoulos VN, Michalopoulos A, Apostolidis S, Harlaftis N (2005) Randomized clinical trial of stapled hemorrhoidectomy vs open with Ligasure for prolapsed piles. Surg Endosc 19(2):235-239

25. Lau PY, Meng WC, Yip AW (2004) Stapled haemorrhoidectomy in Chinese patients: a prospective randomised control study. Hong Kong Med J 10(6):373-377

26. Chung CC, Cheung HY, Chan ES, Kwok SY, Li MK (2005) Stapled hemorrhoidopexy vs. Harmonic Scalpel hemorrhoidectomy: a randomized trial. Dis Colon Rectum. 48(6):1213-1219

27. Gravie JF, Lehur PA, Huten N, Papillon M, Fantoli M, Descottes $B$ et al (2005) Stapled hemorrhoidopexy versus milligan-morgan hemorrhoidectomy: a prospective, randomized, multicenter trial with 2-year postoperative follow up. Ann Surg 242(1):29-35

28. Picchio M, Palimento D, Attanasio U, Renda A (2006) Stapled vs open hemorrhoidectomy: long-term outcome of a randomized controlled trial. Int J Colorectal Dis 21(7):668-669
29. Martinsons A, Narbuts Z, Brunenieks I, Pavars M, Lebedkovs S, Gardovskis J (2007) A comparison of quality of life and postoperative results from combined PPH and conventional haemorrhoidectomy in different cases of haemorrhoidal disease. Colorectal Dis 9(5):423-429

30. Ganio E, Altomare DF, Milito G, Gabrielli F, Canuti S (2007) Long-term outcome of a multicentre randomized clinical trial of stapled haemorrhoidopexy versus Milligan-Morgan haemorrhoidectomy. Br J Surg 94(8):1033-1037

31. Lai HJ, Jao SW, Su CC, Lee MC, Kang JC (2007) Stapled hemorrhoidectomy versus conventional excision hemorrhoidectomy for acute hemorrhoidal crisis. J Gastrointest Surg 11(12):1654-1661

32. Wong JC, Chung CC, Yau KK, Cheung HY, Wong DC, Chan OC et al (2008) Stapled technique for acute thrombosed hemorrhoids: a randomized, controlled trial with long-term results. Dis Colon Rectum 51(4):397-403

33. Stolfi VM, Sileri P, Micossi C, Carbonaro I, Venza M, Gentileschi $P$ et al (2008) Treatment of hemorrhoids in day surgery: stapled hemorrhoidopexy vs Milligan-Morgan hemorrhoidectomy. J Gastrointest Surg 12(5):795-801

34. Leventoglu S, Mentes BB, Akin M, Oguz M (2008) Haemorrhoidectomy with electrocautery or ultrashears and stapled haemorrhoidopexy. ANZ J Surg 78(5):389-393

35. Kraemer M, Parulava T, Roblick M, Duschka L, Muller-Lobeck H (2005) Prospective, randomized study: proximate PPH stapler vs. LigaSure for hemorrhoidal surgery. Dis Colon Rectum. 48(8):1517-1522

36. Nystrom PO, Qvist N, Raahave D, Lindsey I, Mortensen N (2010) Stapled or Open Pile Procedure trial study g. Randomized clinical trial of symptom control after stapled anopexy or diathermy excision for haemorrhoid prolapse. Br J Surg. 97(2):167-176

37. Ammaturo C, Tufano A, Spiniello E, Sodano B, Iervolino EM, Brillantino A et al (2012) Stapled haemorrhoidopexy vs. Milligan-Morgan haemorrhoidectomy for grade III haemorrhoids: a randomized clinical trial. G Chir. 33(10):346-351

38. Arslani N, Patrlj L, Rajkovic Z, Papes D, Altarac S (2012) A randomized clinical trial comparing Ligasure versus stapled hemorrhoidectomy. Surg Laparosc Endosc Percutan Tech 22(1):58-61

39. Kim JS, Vashist YK, Thieltges S, Zehler O, Gawad KA, Yekebas EF et al (2013) Stapled hemorrhoidopexy versus Milligan-Morgan hemorrhoidectomy in circumferential third-degree hemorrhoids: long-term results of a randomized controlled trial. J Gastrointest Surg 17(7):1292-1298

40. Wang ZG, Zhang Y, Zeng XD, Zhang TH, Zhu QD, Liu DL et al (2015) Clinical observations on the treatment of prolapsing hemorrhoids with tissue selecting therapy. World J Gastroenterol 21(8):2490-2496

41. Ripetti V, La Vaccara V, Greco S, Arullani A (2015) A randomized trial comparing stapled rectal mucosectomy versus open and semiclosed hemorrhoidectomy. Dis Colon Rectum 58(11):1083-1090

42. Bilgin Y, Hot S, Barlas IS, Akan A, Eryavuz Y (2015) Short- and long-term results of harmonic scalpel hemorrhoidectomy versus stapler hemorrhoidopexy in treatment of hemorrhoidal disease. Asian J Surg 38(4):214-219

43. Watson AJ, Hudson J, Wood J, Kilonzo M, Brown SR, McDonald A et al (2016) Comparison of stapled haemorrhoidopexy with traditional excisional surgery for haemorrhoidal disease (eTHoS): a pragmatic, multicentre, randomised controlled trial. Lancet 388(10058):2375-2385

44. He YH, Tang ZJ, Xu XT, Huang DQ, Zhang LS, Tang QZ et al (2017) A randomized multicenter clinical trial of rph with the simplified milligan-morgan hemorrhoidectomy in the treatment of mixed hemorrhoids. Surg Innov 24(6):574-581

45. Giarratano G, Toscana E, Toscana C, Petrella G, Shalaby M, Sileri P (2018) Transanal hemorrhoidal dearterialization versus 
stapled hemorrhoidopexy: long-term follow-up of a prospective randomized study. Surg Innov 25(3):236-241

46. Lehur PA, Didnee AS, Faucheron JL, Meurette G, Zerbib P, Siproudhis L et al (2016) Cost-effectiveness of new surgical treatments for hemorrhoidal disease: a multicentre randomized controlled trial comparing transanal doppler-guided hemorrhoidal artery ligation with mucopexy and circular stapled hemorrhoidopexy. Ann Surg 264(5):710-716

47. Leung ALH, Cheung TPP, Tung K, Tsang YP, Cheung H, Lau $\mathrm{CW}$ et al (2017) A prospective randomized controlled trial evaluating the short-term outcomes of transanal hemorrhoidal dearterialization versus tissue-selecting technique. Tech Coloproctol 21(9):737-743

48. Longo A, (eds). Treatment of hemorrhoidal disease by reduction of mucosa and hemorrhoidal prolapse with a circular stapler suturing device: a new procedure. In: Proceedings of the 6th World Congress of Endoscopic Surgery, Rome; 1998.

49. Cohen Z (1985) Symposium on outpatient anorectal procedures. Alternatives to surgical hemorrhoidectomy. Can J Surg. 28(3):230-231

50. Goulimaris I, Kanellos I, Christoforidis E, Mantzoros I, Odisseos C, Betsis D (2002) Stapled haemorrhoidectomy compared with Milligan-Morgan excision for the treatment of prolapsing haemorrhoids: a prospective study. Eur J Surg 168(11):621-625

51. Thaha MA, Irvine LA, Steele RJ, Campbell KL (2005) Postdefaecation pain syndrome after circular stapled anopexy is abolished by oral nifedipine. Br J Surg 92(2):208-210
52. McCloud JM, Jameson JS, Scott AN (2006) Life-threatening sepsis following treatment for haemorrhoids: a systematic review. Colorectal Dis 8(9):748-755

53. Sturiale A, Cafaro D, Fabiani B, Ferro U, Naldini G (2018) Rectal perforation after Doppler-guided hemorrhoidal dearterialization treated with diverting sigmoidostomy. Tech Coloproctol 22(7):553-554

54. Gallo G, Podzemny V, Pescatori M (2016) Intestinal obstruction requiring fecal diversion due to rectal hematoma following a hemorrhoid laser procedure (HeLP). Tech Coloproctol 20(7):507-508

55. Folie P, Wolff K, Marti L, Fretz C, Lange J, Hetzer FH (2010) Rectal pocket syndrome after stapled haemorrhoidopexy. Chirurg 81(1):61-63

56. Naldini G (2011) Serious unconventional complications of surgery with stapler for haemorrhoidal prolapse and obstructed defaecation because of rectocoele and rectal intussusception. Colorectal Dis 13(3):323-327

57. Reenen M JB. EQ-5D-5L User guide-basic information on how to use the EQ-5D-5L instrument EQ-5D. 2015;2.1.

Publisher's Note Springer Nature remains neutral with regard to jurisdictional claims in published maps and institutional affiliations. 